\title{
Solvent-induced chirality switching in the enantioseparation of halogen-substituted mandelic acids: Structural effects on molecular packing
}

Koichi Kodama*, Kozue Kawasaki, Meng Yi, Kaguya Tsukamoto, Hiroaki Shitara and Takuji

Hirose

Graduate School of Science and Engineering, Saitama University

255 Shimo-ohkubo, Sakura-ku, Saitama City, Saitama 338-8570, Japan

Table of Contents

Figures S1-S9 and Tables S1-S2

S2-S16

Experimental detail

S17-S20

References

S20 


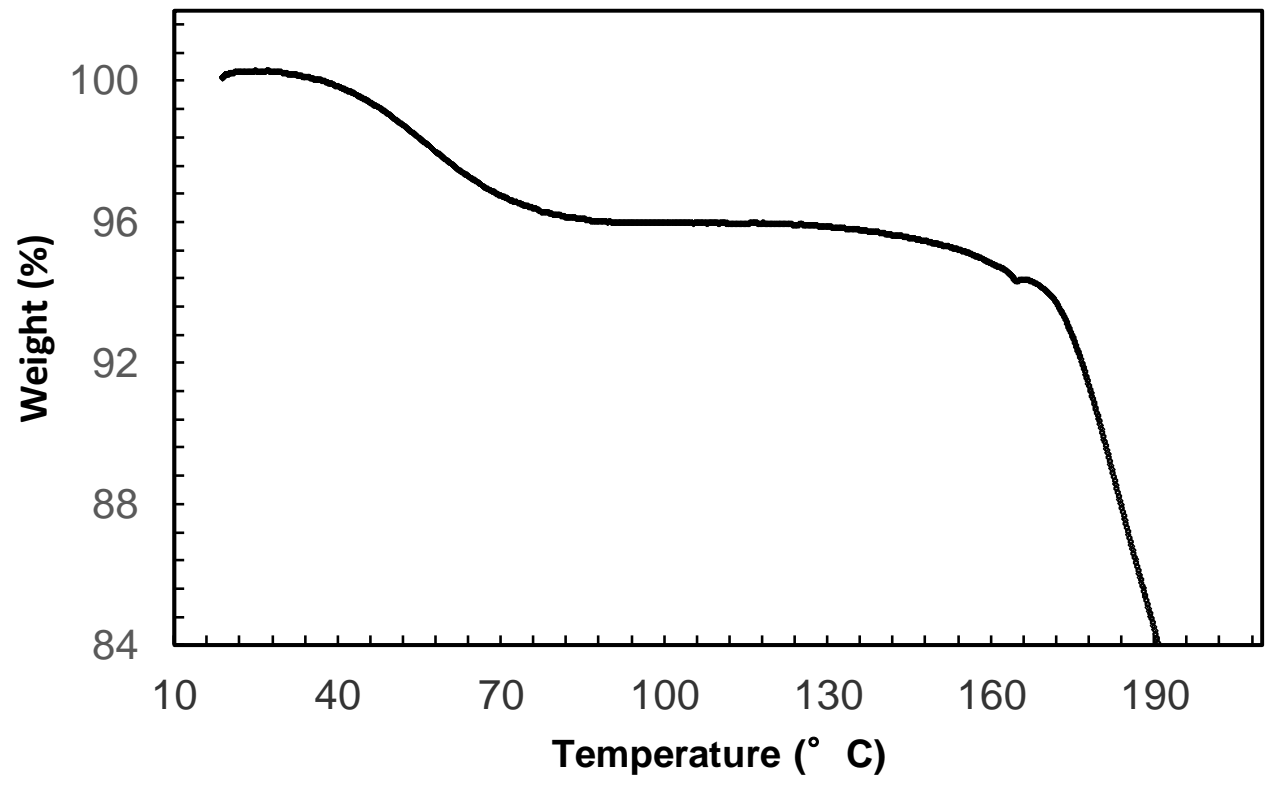

Figure S1. TGA chart of $(S)$ - $p$-Br-MA·(+)-ADPE salt (enantiomer of the $(R)-p$-Br-MA·(-)-ADPE salt) crystallized from water. Heating rate was $10{ }^{\circ} \mathrm{C} / \mathrm{min}$. 

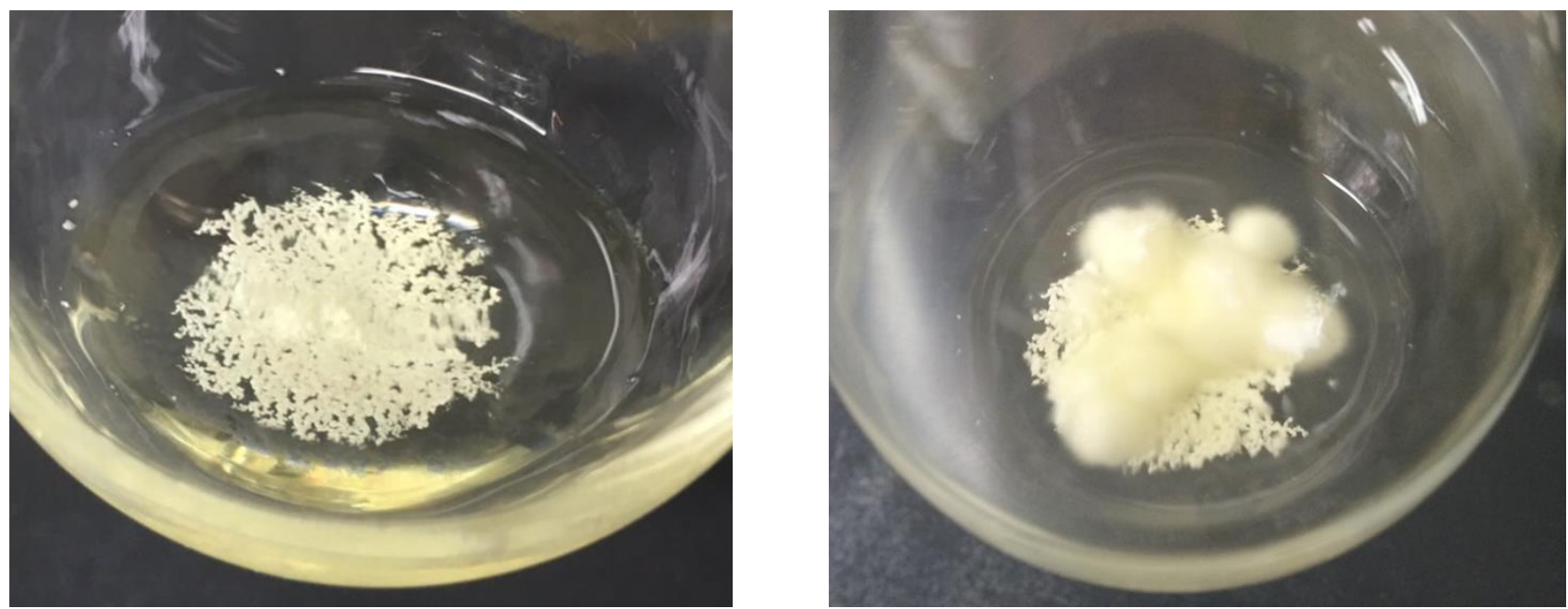

Figure S2. Photographs of the deposited solid in the enantioseparation of $p$-F-MA with (-)-ADPE in acetone. The hot solution was stood at room temperature for about $1 \mathrm{~h}$ (left) and $10 \mathrm{~h}$ (right). 
a)

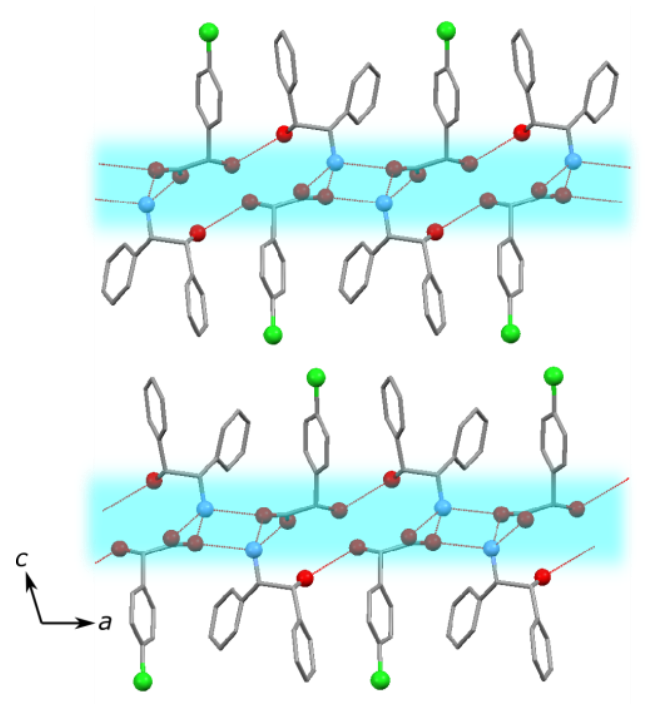

b)
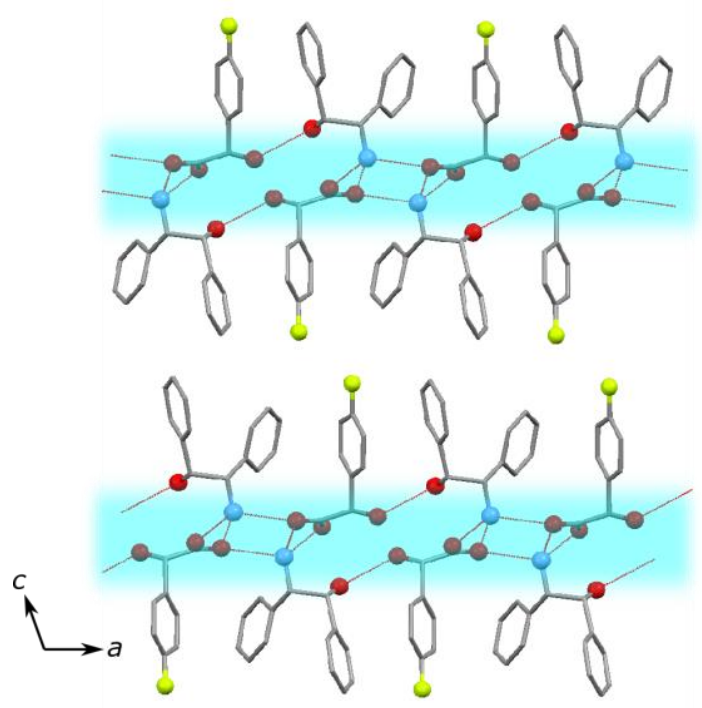

Figure S3. Crystal structures of less-soluble diastereomeric salts a) (S)-p-Cl-MA-(-)-ADPE, and b) (S)-p-F-MA-(-)-ADPE. Oxygen and nitrogen atoms are represented with red and blue balls. Halogen atoms are represented with yellow to green balls. The dotted lines show hydrogen bonds. 


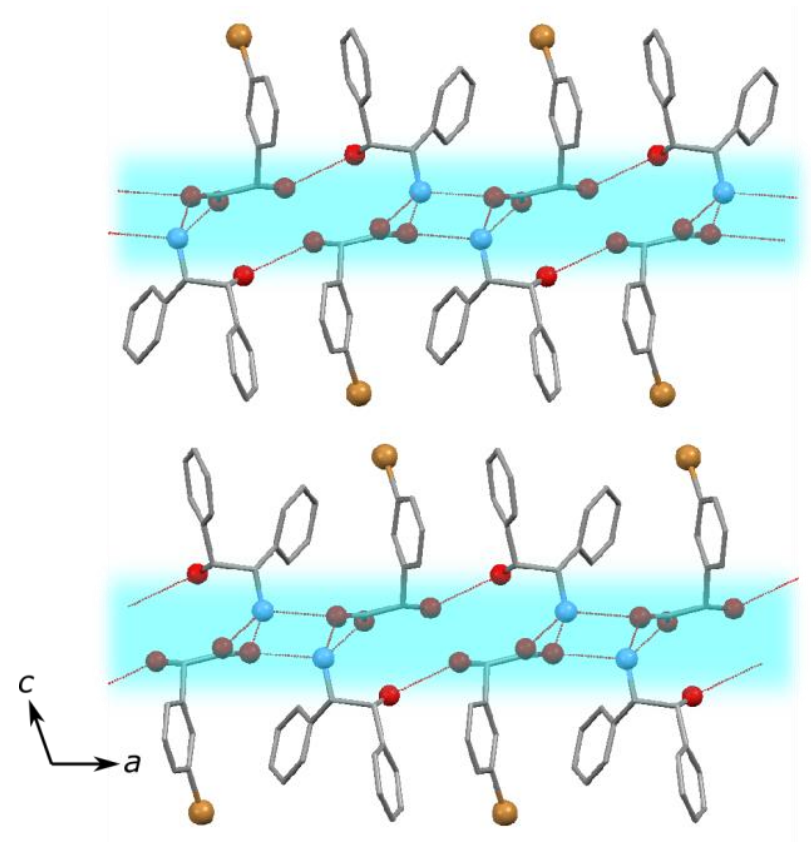

Figure S4. Crystal structure of less-soluble (S)-m-Br-MA-(-)-ADPE salt prepared from chloroform solution. Oxygen and nitrogen atoms are represented with red and blue balls. Bromine atoms are represented with green balls. The dotted lines show hydrogen bonds. 


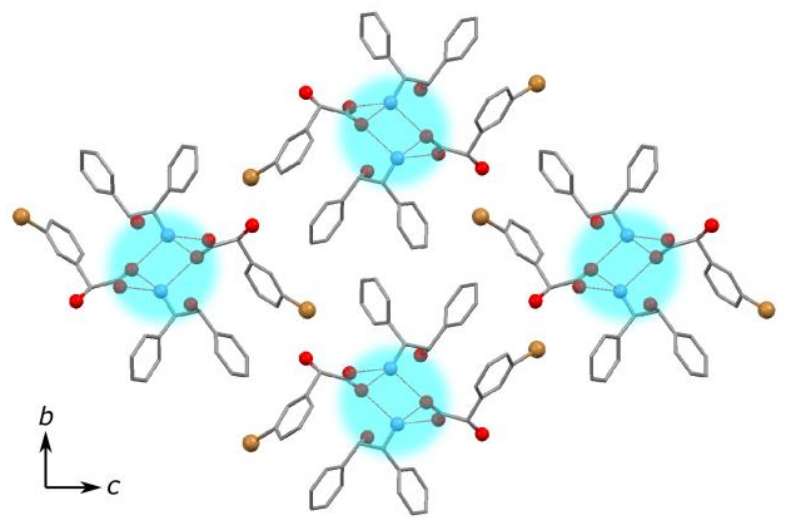

Figure S5. Crystal structure of more-soluble $(S)$-m-Br-MA-(+)-ADPE salt prepared from chloroform solution. Oxygen and nitrogen atoms are represented with red and blue balls. Bromine atoms are represented with green balls. The dotted lines show hydrogen bonds. 


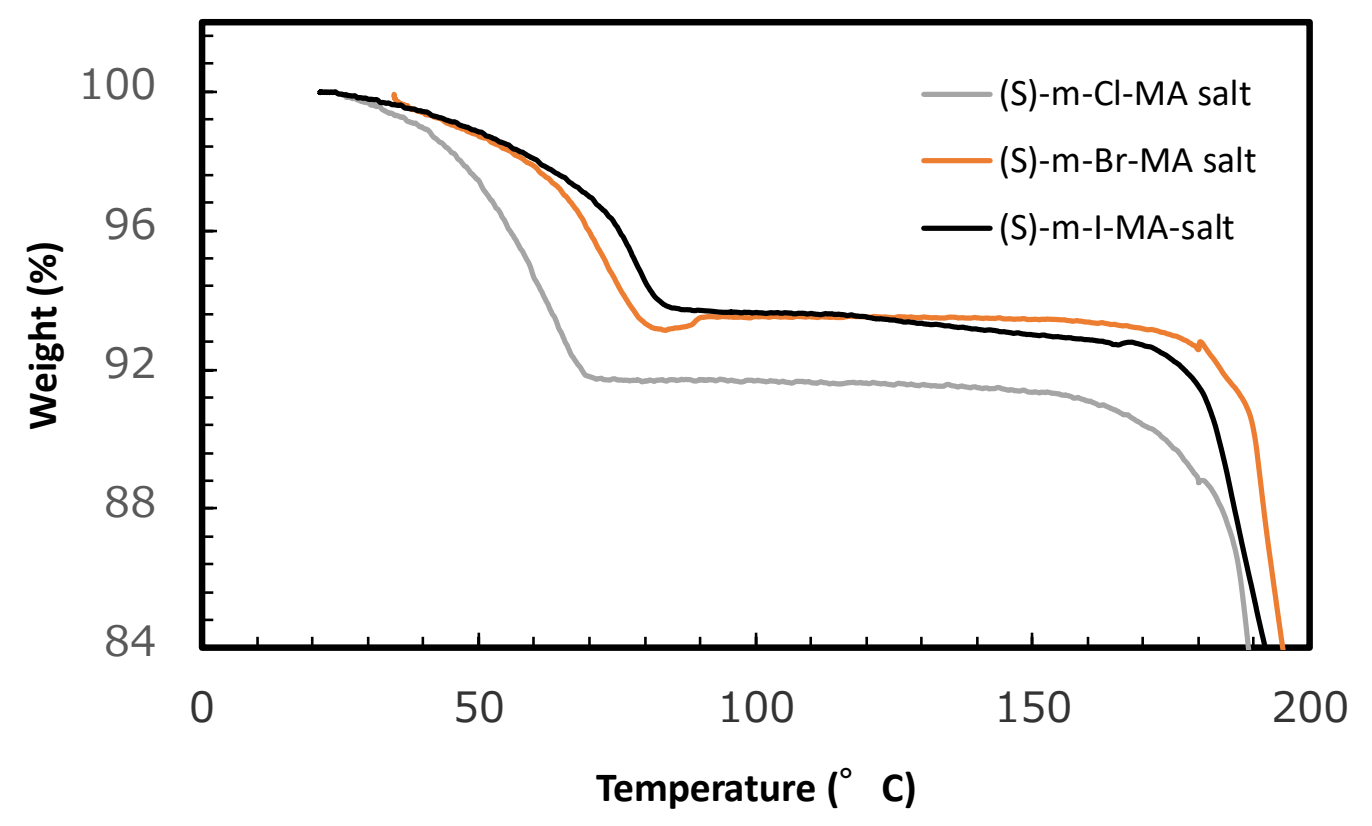

Figure S6. TGA charts of (S)-m-X-MA-(-)-ADPE salt $(\mathrm{X}=\mathrm{Cl}, \mathrm{Br}, \mathrm{I})$ crystallized from water. Heating rate was $10{ }^{\circ} \mathrm{C} / \mathrm{min}$. 


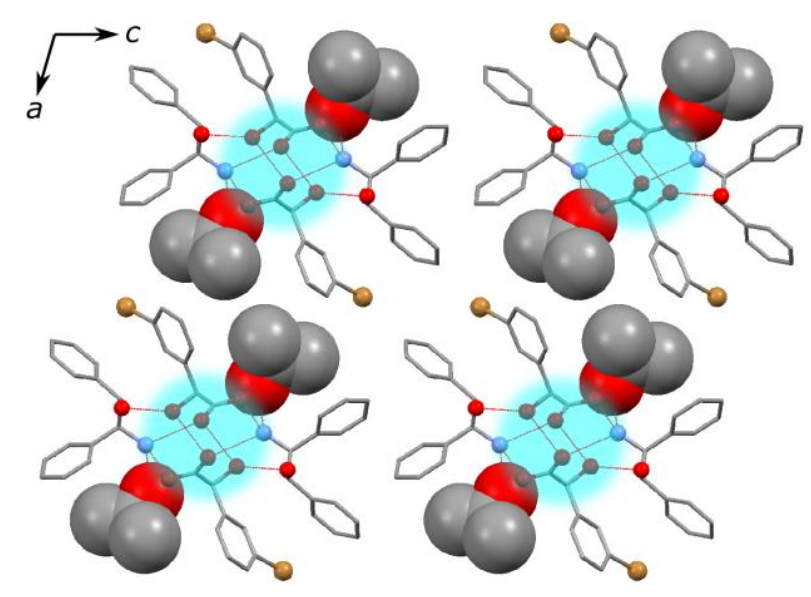

Figure S7. Crystal structure of less-soluble $(S)$ - $m$-Br-MA-(-)-ADPE- $i$-PrOH salt prepared from 2-propanol solution. Oxygen and nitrogen atoms are represented with red and blue balls. Bromine atoms are represented with green balls. The dotted lines show hydrogen bonds. 


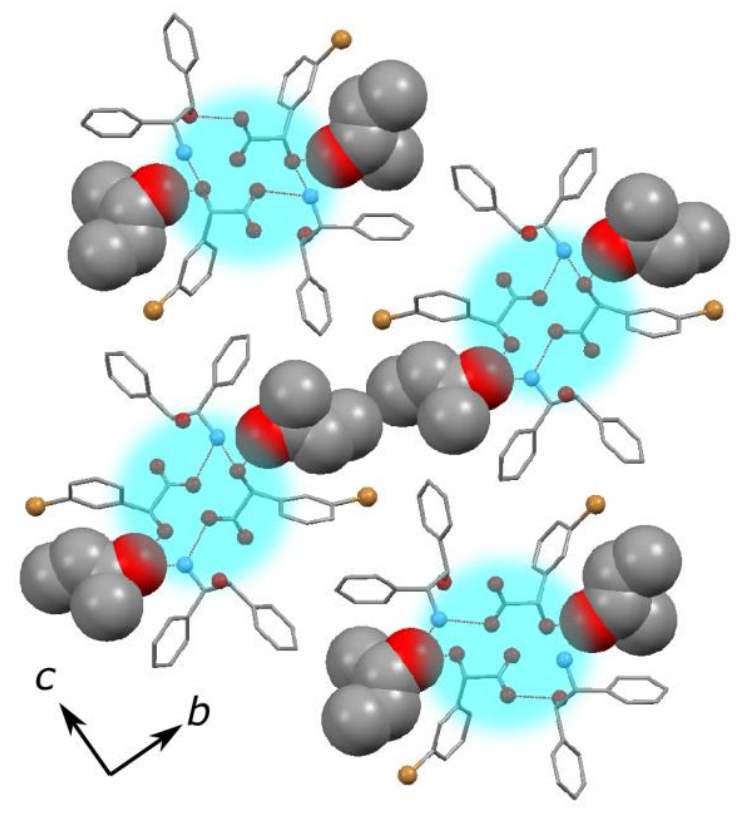

Figure S8. Crystal structure of $(S)$ - $m$-Br-MA-(+)-ADPE- $s$-BuOH salt prepared from 2-butanol solution. Oxygen and nitrogen atoms are represented with red and blue balls. Bromine atoms are represented with green balls. The dotted lines show hydrogen bonds. 


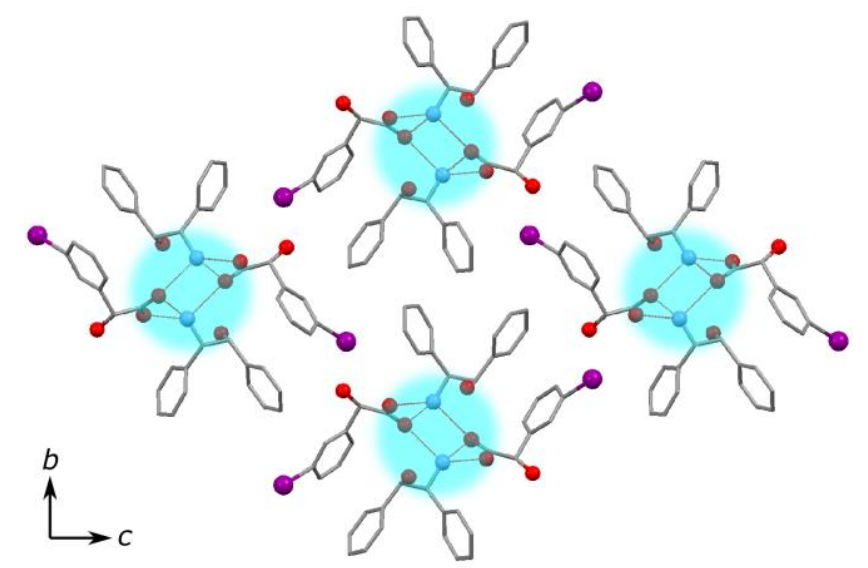

Figure S9. Crystal structure of more-soluble $(R)$-m-I-MA-(-)-ADPE salt prepared from toluene solution. Oxygen and nitrogen atoms are represented with red and blue balls. Iodine atoms are represented with purple balls. The dotted lines show hydrogen bonds. 
Table S1. Summary of crystallographic data reported in this study.

\begin{tabular}{|c|c|c|c|c|c|}
\hline & $\begin{array}{c}(S)-\mathrm{p}-\mathrm{F}-\mathrm{MA} \cdot(1 R, 2 S)- \\
\text { ADPE }\end{array}$ & $\begin{array}{c}(S)-\mathrm{p}-\mathrm{Cl}-\mathrm{MA} \cdot(1 R, 2 S)- \\
\text { ADPE }\end{array}$ & $\begin{array}{c}(S)-\mathrm{p}-\mathrm{Br}-\mathrm{MA} \cdot(1 R, 2 S)- \\
\text { ADPE }\end{array}$ & $(S)-M A \cdot(1 R, 2 S)-A D P E$ & $\begin{array}{c}(S)-\mathrm{m}-\mathrm{Cl}-\mathrm{MA} \cdot(1 R, 2 S)- \\
\text { ADPE }\end{array}$ \\
\hline empirical formula & $\left(\mathrm{C}_{8} \mathrm{H}_{6} \mathrm{O}_{3} \mathrm{~F}\right)^{-} \cdot\left(\mathrm{C}_{14} \mathrm{H}_{16} \mathrm{NO}\right)^{+}$ & $\left(\mathrm{C}_{8} \mathrm{H}_{6} \mathrm{O}_{3} \mathrm{Cl}\right)^{-} \cdot\left(\mathrm{C}_{14} \mathrm{H}_{16} \mathrm{NO}\right)^{+}$ & $\left(\mathrm{C}_{8} \mathrm{H}_{6} \mathrm{O}_{3} \mathrm{Br}\right)^{-} \cdot\left(\mathrm{C}_{14} \mathrm{H}_{16} \mathrm{NO}\right)^{+}$ & $\left(\mathrm{C}_{8} \mathrm{H}_{7} \mathrm{O}_{3}\right)^{-} \cdot\left(\mathrm{C}_{14} \mathrm{H}_{16} \mathrm{NO}\right)^{+}$ & $\left(\mathrm{C}_{8} \mathrm{H}_{6} \mathrm{O}_{3} \mathrm{Cl}\right)^{-} \cdot\left(\mathrm{C}_{14} \mathrm{H}_{16} \mathrm{NO}\right)^{+}$ \\
\hline formula weight & 383.4 & 399.85 & 444.31 & 365.41 & 399.85 \\
\hline temperature $(\mathrm{K})$ & 150 & 150 & 150 & 150 & 150 \\
\hline crystal size (mm) & $0.66 \times 0.15 \times 0.03$ & $0.60 \times 0.08 \times 0.03$ & $0.92 \times 0.32 \times 0.05$ & $0.45 \times 0.02 \times 0.01$ & $0.45 \times 0.02 \times 0.01$ \\
\hline crystal system & monoclinic & monoclinic & monoclinic & monoclinic & monoclinic \\
\hline space group & $P 2_{1}$ & $P 2_{1}$ & $P 2_{1}$ & $C 2$ & $P 2_{1}$ \\
\hline$a(\AA)$ & $10.740(2)$ & $10.675(11)$ & $10.615(5)$ & $30.206(6)$ & $10.760(2)$ \\
\hline$b(\AA)$ & $6.0284(13)$ & $6.069(6)$ & $6.262(3)$ & $5.9102(11)$ & $6.3327(13)$ \\
\hline$c(\AA)$ & $16.127(4)$ & $16.123(17)$ & $15.743(8)$ & $10.554(2)$ & $15.567(3)$ \\
\hline$\alpha\left(^{\circ}\right)$ & 90 & 90 & 90 & 90 & 90 \\
\hline$\beta\left(^{\circ}\right)$ & $109.405(2)$ & $107.088(12)$ & $106.458(5)$ & $97.439(2)$ & $109.277(3)$ \\
\hline$\gamma\left({ }^{\circ}\right)$ & 90 & 90 & 90 & 90 & 90 \\
\hline$V\left(\AA^{3}\right)$ & $984.9(4)$ & $998.4(18)$ & $1003.7(9)$ & $1868.3(6)$ & $1001.2(4)$ \\
\hline$Z$ & 2 & 2 & 2 & 4 & 2 \\
\hline$D c\left(\mathrm{~g} / \mathrm{cm}^{3}\right)$ & 1.293 & 1.330 & 1.470 & 1.299 & 1.326 \\
\hline$\mu\left(\mathrm{Mo}_{\mathrm{Ka}}\right)\left(\mathrm{mm}^{-1}\right)$ & 0.095 & 0.219 & 2.076 & 0.089 & 0.219 \\
\hline$\theta_{\min / \max }\left({ }^{\circ}\right)$ & $1.339 / 24.985$ & $1.321 / 25.000$ & $1.349 / 24.999$ & $1.360 / 25.000$ & $1.386 / 24.996$ \\
\hline$R 1\left[F_{0}>2 \sigma\left(F_{0}\right)\right]$ & 0.1035 & 0.1179 & 0.0735 & 0.0716 & 0.0669 \\
\hline$w R 2\left(\right.$ all $\left.F_{0}^{2}\right)$ & 0.273 & 0.2937 & 0.1874 & 0.178 & 0.1625 \\
\hline GOF & 1.095 & 1.154 & 1.061 & 1.01 & 0.944 \\
\hline Flack parameter & $0.0(10)$ & $-0.1(3)$ & $0.01(2)$ & $-9.8(10)$ & $-0.1(2)$ \\
\hline measured reflns & 4630 & 4546 & 4650 & 4460 & 4809 \\
\hline independent reflns & 3007 & 3085 & 3217 & 2977 & 3292 \\
\hline observed reflns & 2893 & 2263 & 2989 & 2540 & 2252 \\
\hline reflns used & 3007 & 3085 & 3217 & 2977 & 3292 \\
\hline parameters & 261 & 253 & 255 & 244 & 270 \\
\hline CCDC number & 1913091 & 1913092 & 1913093 & 1913094 & 1913095 \\
\hline
\end{tabular}


Table S1. Summary of crystallographic data reported in this study (continued).

\begin{tabular}{|c|c|c|c|c|c|}
\hline & $\begin{array}{c}(S)-\mathrm{m}-\mathrm{Br}-\mathrm{MA} \cdot(1 R, 2 S)- \\
\text { ADPE }\end{array}$ & $\begin{array}{c}(R)-\mathrm{m}-\mathrm{Cl}-\mathrm{MA} \cdot(1 R, 2 S)- \\
\text { ADPE }\end{array}$ & $\begin{array}{c}(S)-\mathrm{m}-\mathrm{Br}-\mathrm{MA} \cdot(1 S, 2 R)- \\
\text { ADPE }\end{array}$ & $\begin{array}{c}(S)-\mathrm{m}-\mathrm{Cl}-\mathrm{MA} \cdot(1 R, 2 S)- \\
\text { ADPE } \cdot 2 \text {-propanol }\end{array}$ & $\begin{array}{c}(S)-\mathrm{m}-\mathrm{Br}-\mathrm{MA} \cdot(1 R, 2 S)- \\
\text { ADPE } \cdot \text { 2-propanol }\end{array}$ \\
\hline empirical formula & $\left(\mathrm{C}_{8} \mathrm{H}_{6} \mathrm{O}_{3} \mathrm{Br}\right)^{-} \cdot\left(\mathrm{C}_{14} \mathrm{H}_{16} \mathrm{NO}\right)^{+}$ & $\left(\mathrm{C}_{8} \mathrm{H}_{6} \mathrm{O}_{3} \mathrm{Cl}\right)^{-} \cdot\left(\mathrm{C}_{14} \mathrm{H}_{16} \mathrm{NO}\right)^{+}$ & $\left(\mathrm{C}_{8} \mathrm{H}_{6} \mathrm{O}_{3} \mathrm{Br}\right)^{-} \cdot\left(\mathrm{C}_{14} \mathrm{H}_{16} \mathrm{NO}\right)^{+}$ & $\begin{array}{l}\left(\mathrm{C}_{8} \mathrm{H}_{6} \mathrm{O}_{3} \mathrm{Cl}\right)^{-} \cdot\left(\mathrm{C}_{14} \mathrm{H}_{16} \mathrm{NO}\right)^{+} \cdot \\
\left(\mathrm{C}_{3} \mathrm{H}_{8} \mathrm{O}\right)\end{array}$ & $\begin{array}{l}\left(\mathrm{C}_{8} \mathrm{H}_{6} \mathrm{O}_{3} \mathrm{Br}\right)^{-} \cdot\left(\mathrm{C}_{14} \mathrm{H}_{16} \mathrm{NO}\right)^{+} \\
\cdot\left(\mathrm{C}_{3} \mathrm{H}_{8} \mathrm{O}\right)\end{array}$ \\
\hline formula weight & 444.31 & 399.85 & 444.31 & 459.95 & 504.41 \\
\hline temperature $(\mathrm{K})$ & 150 & 150 & 150 & 150 & 150 \\
\hline crystal size (mm) & $0.50 \times 0.02 \times 0.01$ & $0.48 \times 0.02 \times 0.01$ & $0.25 \times 0.03 \times 0.02$ & $0.21 \times 0.02 \times 0.01$ & $0.44 \times 0.03 \times 0.03$ \\
\hline crystal system & monoclinic & orthorhombic & orthorhombic & monoclinic & monoclinic \\
\hline space group & $P 2_{1}$ & $P 2_{1} 2_{1} 2_{1}$ & $P 2_{1} 2_{1} 2_{1}$ & $C 2$ & $C 2$ \\
\hline$a(\AA)$ & $10.785(3)$ & $5.6070(9)$ & $5.6728(13)$ & $26.10(2)$ & $26.377(9)$ \\
\hline$b(\AA)$ & $6.2318(14)$ & 13.999(2) & $14.074(3)$ & $5.801(5)$ & $5.8418(19)$ \\
\hline$c(\AA)$ & $15.842(4)$ & $24.658(4)$ & $24.467(6)$ & $16.174(13)$ & $16.368(5)$ \\
\hline$\alpha\left(^{\circ}\right)$ & 90 & 90 & 90 & 90 & 90 \\
\hline$\beta\left(^{\circ}\right)$ & $109.254(3)$ & 90 & 90 & $106.512(10)$ & $106.204(4)$ \\
\hline$\gamma\left({ }^{\circ}\right)$ & 90 & 90 & 90 & 90 & 90 \\
\hline$V\left(\AA^{3}\right)$ & $1005.2(4)$ & 1935.5(5) & $1953.4(8)$ & $2348(3)$ & $2421.9(14)$ \\
\hline$Z$ & 2 & 4 & 4 & 4 & 4 \\
\hline$D c\left(\mathrm{~g} / \mathrm{cm}^{3}\right)$ & 1.468 & 1.372 & 1.511 & 1.301 & 1.383 \\
\hline$\mu\left(\mathrm{Mo}_{\mathrm{Ka}}\right)\left(\mathrm{mm}^{-1}\right)$ & 2.072 & 0.226 & 2.133 & 0.199 & 1.732 \\
\hline$\theta_{\min / \max }\left({ }^{\circ}\right)$ & $1.362 / 24.990$ & $1.652 / 25.000$ & $1.665 / 24.996$ & $1.313 / 24.999$ & $1.296 / 25.000$ \\
\hline$R 1\left[F_{0}>2 \sigma\left(F_{0}\right)\right]$ & 0.0982 & 0.0607 & 0.0450 & 0.0940 & 0.0602 \\
\hline$w R 2\left(\right.$ all $\left.F_{0}^{2}\right)$ & 0.2418 & 0.1434 & 0.1135 & 0.2273 & 0.1377 \\
\hline GOF & 0.980 & 0.929 & 0.948 & 0.902 & 0.891 \\
\hline Flack parameter & $-0.01(3)$ & $0.02(9)$ & $-0.001(12)$ & $-0.3(3)$ & $0.00(2)$ \\
\hline measured reflns & 4758 & 9286 & 9378 & 5487 & 5824 \\
\hline independent reflns & 3196 & 3414 & 3436 & 3835 & 3678 \\
\hline observed reflns & 2268 & 2764 & 2958 & 1793 & 2355 \\
\hline reflns used & 3196 & 3414 & 3436 & 3835 & 3678 \\
\hline parameters & 267 & 273 & 273 & 292 & 294 \\
\hline CCDC number & 1913096 & 1913097 & 1913098 & 1913099 & 1913100 \\
\hline
\end{tabular}


Table S1. Summary of crystallographic data reported in this study (continued).

\begin{tabular}{|c|c|c|c|c|c|}
\hline & $\begin{array}{c}(R)-\mathrm{m}-\mathrm{Cl}-\mathrm{MA} \cdot(1 R, 2 S)- \\
\text { ADPE } \cdot 2 \text {-butanol }\end{array}$ & $\begin{array}{c}(S)-\mathrm{m}-\mathrm{Br}-\mathrm{MA} \cdot(1 S, 2 R)- \\
\text { ADPE } \cdot 2 \text {-butanol }\end{array}$ & $\begin{array}{c}(R) \text {-m-I-MA } \cdot(1 R, 2 S)- \\
\text { ADPE } \cdot 1 \text {-butanol }\end{array}$ & $\begin{array}{c}(\mathrm{S})-\mathrm{m}-\mathrm{I}-\mathrm{MA} \cdot(1 R, 2 \mathrm{~S})- \\
\text { ADPE } \cdot 2\left(\mathrm{H}_{2} \mathrm{O}\right)\end{array}$ & $\begin{array}{c}(R)-\mathrm{m}-\mathrm{I}-\mathrm{MA} \cdot(1 R, 2 S)- \\
\text { ADPE }\end{array}$ \\
\hline empirical formula & $\begin{array}{l}\left(\mathrm{C}_{8} \mathrm{H}_{6} \mathrm{O}_{3} \mathrm{Cl}\right)^{-} \cdot\left(\mathrm{C}_{14} \mathrm{H}_{16} \mathrm{NO}\right)^{+} \\
\cdot\left(\mathrm{C}_{4} \mathrm{H}_{10} \mathrm{O}\right)\end{array}$ & $\begin{array}{l}\left(\mathrm{C}_{8} \mathrm{H}_{6} \mathrm{O}_{3} \mathrm{Br}\right)^{-} \cdot\left(\mathrm{C}_{14} \mathrm{H}_{16} \mathrm{NO}\right)^{+} \\
\cdot\left(\mathrm{C}_{4} \mathrm{H}_{10} \mathrm{O}\right)\end{array}$ & $\begin{array}{l}\left(\mathrm{C}_{8} \mathrm{H}_{6} \mathrm{O}_{3} \mathrm{l}\right)^{-} \cdot\left(\mathrm{C}_{14} \mathrm{H}_{16} \mathrm{NO}\right)^{+} \cdot \\
\left(\mathrm{C}_{4} \mathrm{H}_{10} \mathrm{O}\right)\end{array}$ & $\begin{array}{l}\left(\mathrm{C}_{8} \mathrm{H}_{6} \mathrm{O}_{3} \mathrm{l}\right)^{-} \cdot\left(\mathrm{C}_{14} \mathrm{H}_{16} \mathrm{NO}\right)^{+} \cdot \\
2\left(\mathrm{H}_{2} \mathrm{O}\right)\end{array}$ & $\left(\mathrm{C}_{8} \mathrm{H}_{6} \mathrm{O}_{3} \mathrm{l}\right)^{-} \cdot\left(\mathrm{C}_{14} \mathrm{H}_{16} \mathrm{NO}\right)^{+}$ \\
\hline formula weight & 473.97 & 518.43 & 565.42 & 527.34 & 491.30 \\
\hline temperature (K) & 150 & 150 & 150 & 150 & 150 \\
\hline crystal size (mm) & $0.40 \times 0.02 \times 0.02$ & $0.75 \times 0.02 \times 0.01$ & $0.35 \times 0.02 \times 0.02$ & $0.36 \times 0.02 \times 0.02$ & $1.00 \times 0.04 \times 0.03$ \\
\hline crystal system & orthorhombic & orthorhombic & monoclinic & orthorhombic & orthorhombic \\
\hline space group & $P 2_{1} 2_{1} 2_{1}$ & $P 2_{1} 2_{1} 2_{1}$ & $P 2_{1}$ & $P 2_{1} 2_{1} 2$ & $P 2_{1} 2_{1} 2_{1}$ \\
\hline$a(\AA)$ & $5.5803(15)$ & $5.586(3)$ & $13.907(8)$ & $24.310(5)$ & $5.8084(13)$ \\
\hline$b(\AA)$ & $18.221(5)$ & $18.293(9)$ & $5.348(3)$ & $32.200(6)$ & $14.178(3)$ \\
\hline$c(\AA)$ & $24.567(6)$ & $24.714(12)$ & $16.966(9)$ & $5.6265(11)$ & $24.289(5)$ \\
\hline$\alpha\left(^{\circ}\right)$ & 90 & 90 & 90 & 90 & 90 \\
\hline$\beta\left(^{\circ}\right)$ & 90 & 90 & $95.847(8)$ & 90 & 90 \\
\hline$\gamma\left({ }^{\circ}\right)$ & 90 & 90 & 90 & 90 & 90 \\
\hline$V\left(\AA^{3}\right)$ & $2498.0(12)$ & $2525(2)$ & $1255.2(12)$ & $4404.3(15)$ & $2000.2(8)$ \\
\hline$Z$ & 4 & 4 & 2 & 8 & 4 \\
\hline$D c\left(\mathrm{~g} / \mathrm{cm}^{3}\right)$ & 1.26 & 1.364 & 1.496 & 1.591 & 1.631 \\
\hline$\mu\left(\mathrm{Mo}_{\mathrm{Ka}}\right)\left(\mathrm{mm}^{-1}\right)$ & 0.189 & 1.663 & 1.311 & 1.492 & 1.629 \\
\hline$\theta_{\min / \max }\left({ }^{\circ}\right)$ & $1.391 / 24.989$ & $1.385 / 24.990$ & $1.206 / 24.996$ & $1.265 / 24.993$ & $1.663 / 24.990$ \\
\hline$R 1\left[F_{0}>2 \sigma\left(F_{0}\right)\right]$ & 0.0502 & 0.0684 & 0.076 & 0.0704 & 0.0758 \\
\hline$w R 2\left(\right.$ all $\left.F_{0}^{2}\right)$ & 0.1056 & 0.1243 & 0.1373 & 0.1551 & 0.1634 \\
\hline GOF & 0.911 & 0.853 & 0.91 & 0.914 & 1.023 \\
\hline Flack parameter & $-0.04(9)$ & $-0.012(18)$ & $0.01(6)$ & $0.01(4)$ & $-0.02(4)$ \\
\hline measured reflns & 12025 & 12056 & 5935 & 21375 & 9476 \\
\hline independent reflns & 4408 & 4441 & 3583 & 7750 & 3512 \\
\hline observed reflns & 3140 & 2787 & 2315 & 4652 & 3240 \\
\hline reflns used & 4408 & 4441 & 3583 & 7750 & 3512 \\
\hline parameters & 335 & 311 & 301 & 542 & 243 \\
\hline CCDC number & 1913101 & 1913102 & 1913103 & 1913104 & 1913105 \\
\hline
\end{tabular}


Table S2. Hydrogen bond metrics of the crystals reported in this study.

\begin{tabular}{|c|c|c|c|c|c|}
\hline Compound name & $\mathrm{D}-\mathrm{H} \ldots \mathrm{A}$ & $\mathrm{D}-\mathrm{H} / \AA$ & $\mathrm{H}-\mathrm{A} / \AA$ & $D-A / \AA$ & $\angle \mathrm{D}-\mathrm{H} \ldots \mathrm{A} /{ }^{\circ}$ \\
\hline \multicolumn{6}{|c|}{ (S)-p-F-MA · (1R,2S)-ADPE } \\
\hline & $\mathrm{N} 1-\mathrm{H} 20 \ldots \mathrm{O} 3$ & 1.033 & 1.686 & 2.713 & 172.69 \\
\hline & $\mathrm{N} 1-\mathrm{H} 21 \ldots \mathrm{O} 2$ & 1.033 & 1.865 & 2.818 & 151.77 \\
\hline & $\mathrm{N} 1-\mathrm{H} 22 \ldots \mathrm{O} 2$ & 1.033 & 1.733 & 2.746 & 166.04 \\
\hline & O1-H18...O4 & 0.655 & 2.302 & 2.838 & 140.45 \\
\hline \multicolumn{6}{|c|}{ (S)-p-CI-MA · (1R,2S)-ADPE } \\
\hline & N1-H19...O4 & 1.033 & 1.658 & 2.688 & 174.09 \\
\hline & $\mathrm{N} 1-\mathrm{H} 20 \ldots \mathrm{O} 2$ & 1.033 & 1.860 & 2.814 & 151.93 \\
\hline & $\mathrm{N} 1-\mathrm{H} 21 \ldots \mathrm{O} 2$ & 1.033 & 1.737 & 2.741 & 162.95 \\
\hline & $\mathrm{O} 3-\mathrm{H} 22 \ldots \mathrm{O} 1$ & 0.840 & 2.127 & 2.826 & 140.41 \\
\hline \multicolumn{6}{|c|}{ (S)-p-Br-MA · (1R,2S)-ADPE } \\
\hline & $\mathrm{N} 1-\mathrm{H} 20 \ldots \mathrm{O} 4$ & 1.033 & 1.660 & 2.692 & 176.19 \\
\hline & $\mathrm{N} 1-\mathrm{H} 21 \ldots \mathrm{O} 1$ & 1.032 & 1.744 & 2.738 & 160.40 \\
\hline & $\mathrm{N} 1-\mathrm{H} 22 \ldots \mathrm{O} 1$ & 1.033 & 1.855 & 2.817 & 153.45 \\
\hline & $\mathrm{O} 2-\mathrm{H} 1 \ldots \mathrm{O} 3$ & 0.840 & 1.945 & 2.772 & 168.04 \\
\hline \multirow{2}{*}{\multicolumn{6}{|c|}{ (S)-MA $\cdot(1 R, 2 S)-A D P E$}} \\
\hline & & & & & \\
\hline & $\mathrm{N} 1-\mathrm{H} 21 \ldots \mathrm{O} 3$ & 1.034 & 1.710 & 2.723 & 165.38 \\
\hline & $\mathrm{N} 1-\mathrm{H} 22 \ldots \mathrm{O} 1$ & 1.033 & 1.752 & 2.774 & 169.65 \\
\hline & $\mathrm{N} 1-\mathrm{H} 23 \ldots \mathrm{O} 1$ & 1.033 & 1.879 & 2.859 & 157.16 \\
\hline & $\mathrm{O} 4-\mathrm{H} 11 \ldots \mathrm{O} 2$ & 0.839 & 2.070 & 2.842 & 152.71 \\
\hline \multicolumn{6}{|c|}{ (S)-m-Cl-MA · (1R,2S)-ADPE } \\
\hline & N2-H19...O2 & 0.922 & 1.928 & 2.810 & 159.58 \\
\hline & N2-H20...O2 & 0.937 & 1.931 & 2.748 & 144.48 \\
\hline & $\mathrm{N} 2-\mathrm{H} 21 \ldots \mathrm{O} 3$ & 0.983 & 1.754 & 2.719 & 166.11 \\
\hline & O1-H18...O4 & 0.745 & 2.075 & 2.785 & 159.39 \\
\hline \multicolumn{6}{|c|}{ (S)-m-Br-MA · (1R,2S)-ADPE } \\
\hline & $\mathrm{N} 1-\mathrm{H} 18 \ldots \mathrm{O} 4$ & 0.984 & 1.736 & 2.711 & 170.62 \\
\hline & N1-H19...O2 & 1.038 & 1.797 & 2.741 & 149.17 \\
\hline & $\mathrm{N} 1-\mathrm{H} 20 \ldots \mathrm{O} 2$ & 0.794 & 2.085 & 2.832 & 156.69 \\
\hline & $\mathrm{O} 1-\mathrm{H} 22 \ldots \mathrm{O} 5$ & 0.840 & 1.988 & 2.812 & 166.45 \\
\hline \multicolumn{6}{|c|}{ (R)-m-Cl-MA · (1R,2S)-ADPE } \\
\hline & $\mathrm{N} 1-\mathrm{H} 18 \ldots \mathrm{O} 3$ & 0.768 & 2.008 & 2.772 & 173.28 \\
\hline & N1-H19...O5 & 1.044 & 1.849 & 2.805 & 150.59 \\
\hline & $\mathrm{N} 1-\mathrm{H} 20 \ldots \mathrm{O} 3$ & 1.055 & 1.739 & 2.750 & 159.12 \\
\hline \multicolumn{6}{|c|}{ (S)-m-Br-MA · (1S,2R)-ADPE } \\
\hline & $\mathrm{N} 1-\mathrm{H} 3 \ldots \mathrm{O} 4$ & 0.874 & 1.950 & 2.818 & 171.47 \\
\hline & $\mathrm{N} 1-\mathrm{H} 4 \ldots \mathrm{O} 3$ & 0.886 & 1.940 & 2.755 & 152.26 \\
\hline & $\mathrm{N} 1-\mathrm{H} 5 \ldots \mathrm{O} 3$ & 0.843 & 1.978 & 2.768 & 155.59 \\
\hline
\end{tabular}


Table S2. Hydrogen bond metrics of the crystals reported in this study (continued).

\begin{tabular}{|c|c|c|c|c|c|}
\hline Compound name & D-H...A & $\mathrm{D}-\mathrm{H} / \AA$ & $\mathrm{H}-\mathrm{A} / \AA$ & $D-A / \AA$ & $\angle \mathrm{D}-\mathrm{H} \ldots \mathrm{A} /{ }^{\circ}$ \\
\hline \multicolumn{6}{|c|}{$\begin{array}{l}\text { (S)-m-Cl-MA · (1R,2S)-ADPE } \cdot \\
\text { 2-propanol }\end{array}$} \\
\hline & $\mathrm{N} 1-\mathrm{H} 3 \ldots \mathrm{O} 3$ & 1.034 & 1.834 & 2.820 & 158.20 \\
\hline & N1-H4 ...O5 & 1.033 & 1.696 & 2.704 & 163.94 \\
\hline & $\mathrm{N} 1-\mathrm{H} 5 \ldots \mathrm{O} 4$ & 1.033 & 1.905 & 2.763 & 138.29 \\
\hline & O1-H1...O3 & 0.841 & 1.918 & 2.709 & 156.11 \\
\hline & $\mathrm{O} 2-\mathrm{H} 2 \ldots \mathrm{O} 1$ & 0.839 & 1.872 & 2.684 & 162.36 \\
\hline & O5-H14...O4 & 0.840 & 2.010 & 2.610 & 127.75 \\
\hline \multicolumn{6}{|c|}{$\begin{array}{l}\text { (S)-m-Br-MA } \cdot(1 \mathrm{R}, 2 \mathrm{~S})-\mathrm{ADPE} \cdot \\
\text { 2-propanol }\end{array}$} \\
\hline & $\mathrm{N} 1-\mathrm{H} 28 \ldots \mathrm{O} 4$ & 1.032 & 1.836 & 2.818 & 157.54 \\
\hline & $\mathrm{N} 1-\mathrm{H} 29 \ldots \mathrm{O} 3$ & 1.033 & 1.914 & 2.777 & 138.91 \\
\hline & N1-H30...O5 & 1.033 & 1.699 & 2.705 & 163.29 \\
\hline & $\mathrm{O} 1-\mathrm{H} 1 \ldots \mathrm{O} 4$ & 0.840 & 1.959 & 2.745 & 155.59 \\
\hline & $\mathrm{O} 2-\mathrm{H} 2 \ldots \mathrm{O} 1$ & 0.840 & 1.952 & 2.728 & 153.29 \\
\hline & O5-H8...O3 & 0.840 & 1.932 & 2.653 & 143.30 \\
\hline \multicolumn{6}{|c|}{$\begin{array}{l}\text { (R)-m-Cl-MA · (1R,2S)-ADPE · } \\
\text { 2-butanol }\end{array}$} \\
\hline & $\mathrm{N} 1-\mathrm{H} 36 \ldots \mathrm{O} 2$ & 0.926 & 2.099 & 2.914 & 146.23 \\
\hline & $\mathrm{N} 1-\mathrm{H} 37 \ldots \mathrm{O} 5$ & 0.979 & 1.789 & 2.759 & 170.16 \\
\hline & $\mathrm{N} 1-\mathrm{H} 38 \ldots \mathrm{O} 3$ & 1.005 & 1.732 & 2.702 & 161.14 \\
\hline & O1-H1...O4 & 0.839 & 1.774 & 2.609 & 172.75 \\
\hline & $\mathrm{O} 5-\mathrm{H} 39 \ldots \mathrm{O} 2$ & 0.840 & 2.060 & 2.849 & 156.21 \\
\hline \multicolumn{6}{|c|}{$\begin{array}{l}\text { (S)-m-Br-MA · (1S,2R)-ADPE . } \\
\text { 2-butanol }\end{array}$} \\
\hline & N1-H36...O1 & 1.033 & 1.719 & 2.710 & 159.14 \\
\hline & N1-H37...O5 & 1.034 & 1.763 & 2.761 & 160.90 \\
\hline & N1-H38...O3 & 1.034 & 2.078 & 2.919 & 136.90 \\
\hline & $\mathrm{O} 2-\mathrm{H} 1 \ldots \mathrm{O} 4$ & 0.840 & 1.781 & 2.609 & 168.04 \\
\hline & O5-H40...O3 & 0.840 & 2.066 & 2.848 & 154.62 \\
\hline \multicolumn{6}{|c|}{$\begin{array}{l}\text { (R)-m-I-MA } \cdot(1 R, 2 S)-A D P E \cdot \\
\text { 1-butanol }\end{array}$} \\
\hline & N1-H30...O2 & 1.033 & 1.658 & 2.677 & 167.91 \\
\hline & N1-H31...O5 & 1.033 & 1.979 & 2.836 & 138.46 \\
\hline & $\mathrm{N} 1-\mathrm{H} 32 \ldots \mathrm{O} 1$ & 1.033 & 1.747 & 2.758 & 165.10 \\
\hline & $\mathrm{O} 3-\mathrm{H} 2 \ldots \mathrm{O} 4$ & 0.840 & 1.811 & 2.618 & 160.63 \\
\hline & O1-H33...O5 & 0.840 & 2.124 & 2.899 & 153.32 \\
\hline
\end{tabular}


Table S2. Hydrogen bond metrics of the crystals reported in this study (continued).

\begin{tabular}{|c|c|c|c|c|c|}
\hline Compound name & D-H...A & $\mathrm{D}-\mathrm{H} / \AA$ & $\mathrm{H}-\mathrm{A} / \AA$ & $\mathrm{D}-\mathrm{A} / \AA$ & $\angle \mathrm{D}-\mathrm{H} \ldots \mathrm{A} /{ }^{\circ}$ \\
\hline \multicolumn{6}{|c|}{$\begin{array}{l}\text { (S)-m-I-MA · (1R,2S)-ADPE · } \\
\text { 2(H2O) }\end{array}$} \\
\hline & N1-H1A...O9 & 1.034 & 1.778 & 2.704 & 147.00 \\
\hline & N1-H1B...O8 & 1.033 & 1.851 & 2.815 & 153.65 \\
\hline & N1-H1C...O5 & 1.033 & 1.906 & 2.815 & 144.97 \\
\hline & $\mathrm{N} 2-\mathrm{H} 2 \mathrm{~A} \ldots \mathrm{O} 10$ & 1.033 & 1.982 & 2.909 & 147.86 \\
\hline & N2-H2B...O7 & 1.033 & 1.765 & 2.714 & 150.77 \\
\hline & N2-H2C...O6 & 1.032 & 1.877 & 2.864 & 158.91 \\
\hline & O2-H40...O5 & 0.839 & 2.072 & 2.784 & 142.34 \\
\hline & O4...02 & & & 2.842 & \\
\hline & O5...011 & & & 2.748 & \\
\hline & O6...07 & & & 2.748 & \\
\hline & O6...012 & & & 2.783 & \\
\hline & O8...09 & & & 2.727 & \\
\hline & O8...011 & & & 2.864 & \\
\hline & $011 \ldots 012$ & & & 2.801 & \\
\hline \multicolumn{6}{|l|}{ (R)-m-I-MA $\cdot(1 R, 2 S)-A D P E$} \\
\hline & N1-H20...O1 & 1.033 & 1.738 & 2.761 & 169.93 \\
\hline & $\mathrm{N} 1-\mathrm{H} 21 \ldots \mathrm{O} 1$ & 1.033 & 1.810 & 2.771 & 153.08 \\
\hline & $\mathrm{N} 1-\mathrm{H} 22 \ldots \mathrm{O} 4$ & 1.033 & 1.818 & 2.826 & 164.19 \\
\hline
\end{tabular}




\section{General and Materials}

All the ${ }^{1} \mathrm{H}$ and ${ }^{13} \mathrm{C}$ NMR spectra were recorded on a 300, 400 or $500 \mathrm{MHz}$ spectrometer. IR spectra were reported in reciprocal centimeters. Melting points are uncorrected. Optical rotation values were measured with a polarimeter. All the solvents and $(1 R, 2 S)-(-)$-ADPE were purchased and used as received. The enantiomeric excess of the mandelic acids was determined by chiral HPLC analysis (Daicel Chiralcel OD-3 column $4.6 \times 250 \mathrm{~mm}$ ) with UV detection at $254 \mathrm{~nm}$ after derivatized to the corresponding methyl esters by the reaction with $\mathrm{TMSCHN}_{2}$.

\section{Synthesis and characterization}

Halogen substituted mandelic acids except for 4-fluoromandelic acid were prepared from the corresponding acetophenones by $\alpha$-dibromination followed by hydrolysis under basic condition. ${ }^{1}$ 4-Fluoromandelic acid was synthesized from 4-fluorobenzaldehyde according to the literature procedure. $^{2}$

Procedure for the synthesis of 4-bromomandelic acid (p-Br-MA): 4-Bromoacetophenone (9.96 g, $50.0 \mathrm{mmol})$ was dissolved in acetic acid $(30 \mathrm{~mL})$ and the solution was cooled to $20{ }^{\circ} \mathrm{C}$. To the solution was added dropwise bromine $(16.3 \mathrm{~g}, 102 \mathrm{mmol})$ dissolved in acetic acid $(10 \mathrm{~mL})$ while maintaining the temperature below $40{ }^{\circ} \mathrm{C}$. After stirring for 2 hours at $0{ }^{\circ} \mathrm{C}$, the precipitated solid was filtered and washed with $50 \%$ ethanol and dried under reduced pressure to afford $\alpha, \alpha, 4$-tribromoacetophenone as a white solid (14.0 g, $39.2 \mathrm{mmol}, 79 \%)$. Mp. 93-95 ${ }^{\circ} \mathrm{C} .{ }^{1} \mathrm{H}$ NMR $\left(500 \mathrm{MHz}, \mathrm{CDCl}_{3}\right): \delta(\mathrm{ppm}) 7.97(\mathrm{~d}, J=8.8 \mathrm{~Hz}, 2 \mathrm{H}), 7.66(\mathrm{~d}, J=8.8 \mathrm{~Hz}, 2 \mathrm{H}), 6.59(\mathrm{~s}, 1 \mathrm{H})$. IR $(\mathrm{KBr}): v\left(\mathrm{~cm}^{-1}\right) 3447,3040,1693,1582,1395,1202,1070$.

To the ice-cooled mixture of $\alpha, \alpha, 4$-tribromoacetophenone $(13.9 \mathrm{~g}, 39.0 \mathrm{mmol})$ and water $(15 \mathrm{~mL})$ was added dropwise $\mathrm{NaOH}$ aq. $(19.8 \mathrm{M}, 10 \mathrm{~mL})$ and the mixture was stirred for 3 days below $5{ }^{\circ} \mathrm{C}$. Insoluble solid was filtered off and washed with water. The filtrate was washed with ether $(20 \mathrm{~mL} \times$ 7) and the aqueous phase was acidified with conc. $\mathrm{HCl}$ aq. $(20 \mathrm{~mL})$. The aqueous phase was extracted with ether $(20 \mathrm{~mL} \times 4)$ and the combined organic phase was dried over anhydrous sodium sulfate. After concentration under reduced pressure, the residue was dissolved in methanol and benzylamine ( $4.08 \mathrm{~g}, 38.1 \mathrm{mmol}$ ) was added. After the solvent was removed under reduced pressure, the solid was recrystallized from $80 \%$ ethanol $(51 \mathrm{~mL})$. The obtained salt was added to $1 \mathrm{~N} \mathrm{HCl}$ aq. and extracted with ether $(10 \mathrm{~mL} \times 3)$. The combined organic phase was dried over anhydrous sodium sulfate and the solvent was removed under reduced pressure to give 4-bromomandelic acid (7.40 g, $32.0 \mathrm{mmol}, 83 \%)$ as a white solid. Mp. 118.5-120.0 ${ }^{\circ} \mathrm{C} .{ }^{1} \mathrm{H}$ NMR $\left(300 \mathrm{MHz}, \mathrm{CDCl}_{3}\right): \delta(\mathrm{ppm}) 7.52$ $(\mathrm{d}, J=8.4 \mathrm{~Hz}, 2 \mathrm{H}), 7.34$ (d, $J=8.4 \mathrm{~Hz}, 2 \mathrm{H}), 5.23$ (s, 1H). IR (KBr): $v\left(\mathrm{~cm}^{-1}\right) 3411,2964,1725,1490$, $1260,1189,1058$.

$p$-Cl-MA, $o$-Cl-MA, $m$-Cl-MA, $m$-Br-MA, $m$-I-MA were prepared according to the above procedure. 
$\boldsymbol{\alpha}, \boldsymbol{\alpha}$-dibromo-4-chloroacetophenone. Pale yellow solid. Yield 72\%. Mp. 93-96 ${ }^{\circ} \mathrm{C} .{ }^{1} \mathrm{H}$ NMR $(300$ $\mathrm{MHz}_{\mathrm{CDCl}}$ ): $\delta(\mathrm{ppm}) 8.05(\mathrm{~d}, J=8.9 \mathrm{~Hz}, 2 \mathrm{H}), 7.49$ (d, $\left.J=8.9 \mathrm{~Hz}, 2 \mathrm{H}\right), 6.60(\mathrm{~s}, 1 \mathrm{H}) . \mathrm{IR}(\mathrm{KBr}): v$ $\left(\mathrm{cm}^{-1}\right)$ 3037, 1695, 1587, 1401, 1276, 1205, 1093, 988.

$\boldsymbol{\alpha}, \boldsymbol{\alpha}$-dibromo-2-chloroacetophenone. Purified by silica gel column chromatography (eluent; hexane $\left.: \mathrm{CHCl}_{3}=3: 1\right)$ and obtained as pale yellow oil. Yield $67 \% .{ }^{1} \mathrm{H} \mathrm{NMR}\left(300 \mathrm{MHz}, \mathrm{CDCl}_{3}\right): \delta$ (ppm) 7.65-7.62 (m, 1H), 7.51-7.47 (m, 2H), 7.43-7.37 (m, 1H), $6.79(\mathrm{~s}, 1 \mathrm{H})$. IR (neat): $v\left(\mathrm{~cm}^{-1}\right)$ 3427, 3008, 1722, 1589, 1435, 1198, 1065.

$\boldsymbol{\alpha}, \boldsymbol{\alpha}$-dibromo-3-chloroacetophenone. Purified by silica gel column chromatography (eluent; hexane : $\left.\mathrm{CHCl}_{3}=3: 1\right)$ and obtained as pale yellow oil. Yield $88 \%$. ${ }^{1} \mathrm{H} \mathrm{NMR}\left(500 \mathrm{MHz}, \mathrm{CDCl}_{3}\right): \delta$ $(\mathrm{ppm}) 8.07\left(\mathrm{dd}, J_{1}=J_{2}=1.7 \mathrm{~Hz}, 1 \mathrm{H}\right), 7.98\left(\mathrm{ddd}, J_{1}=7.9 \mathrm{~Hz}, J_{2}=J_{3}=1.7 \mathrm{~Hz}, 1 \mathrm{H}\right), 7.63-7.59(\mathrm{~m}$, $1 \mathrm{H}), 7.46\left(\mathrm{dd}, J_{1}=J_{2}=7.9 \mathrm{~Hz}, 1 \mathrm{H}\right), 6.62(\mathrm{~s}, 1 \mathrm{H})$. IR (neat): $v\left(\mathrm{~cm}^{-1}\right) 3384,3013,1701,1566,1419$, $1254,1069$.

$\alpha, \alpha, 3$-tribromoacetophenone. Purified by silica gel column chromatography (eluent; hexane : $\left.\mathrm{CHCl}_{3}=3: 1\right)$ and obtained as pale yellow oil. Yield 90\%. ${ }^{1} \mathrm{H} \mathrm{NMR}\left(500 \mathrm{MHz}, \mathrm{CDCl}_{3}\right): \delta(\mathrm{ppm})$ $8.21\left(\mathrm{dd}, J_{1}=J_{2}=1.8 \mathrm{~Hz}, 1 \mathrm{H}\right), 8.04-8.01(\mathrm{~m}, 1 \mathrm{H}), 7.78-7.74(\mathrm{~m}, 1 \mathrm{H}), 7.39\left(\mathrm{dd}, J_{1}=J_{2}=7.8 \mathrm{~Hz}, 1 \mathrm{H}\right)$, 6.60 (s, 1H). IR (neat): $v\left(\mathrm{~cm}^{-1}\right)$ 3384, 3013, 1702, 1566, 1419, 1254, 1192, 1069.

$\boldsymbol{\alpha}, \boldsymbol{\alpha}$-dibromo-3-iodoacetophenone. Purified by silica gel column chromatography (eluent; hexane : $\left.\mathrm{CHCl}_{3}=1: 1\right)$ and obtained as pale yellow oil. Yield 85\%. ${ }^{1} \mathrm{H} \mathrm{NMR}\left(400 \mathrm{MHz}, \mathrm{CDCl}_{3}\right): \delta(\mathrm{ppm})$ $8.40\left(\mathrm{dd}, J_{1}=J_{2}=1.6 \mathrm{~Hz}, 1 \mathrm{H}\right), 8.07-8.05(\mathrm{~m}, 1 \mathrm{H}), 7.98-7.95(\mathrm{~m}, 1 \mathrm{H}), 7.25\left(\mathrm{dd}, J_{1}=J_{2}=8.0 \mathrm{~Hz}, 1 \mathrm{H}\right)$, $6.60(\mathrm{~s}, 1 \mathrm{H}) .{ }^{13} \mathrm{C} \mathrm{NMR}\left(100 \mathrm{MHz}, \mathrm{CDCl}_{3}\right): \delta(\mathrm{ppm}) 184.6,143.1,138.5,132.6,130.4,128.8,94.4$, 39.0. IR (neat): $v\left(\mathrm{~cm}^{-1}\right)$ 1699, 1560, 1414, 1254, 1190, 988, 806, 672, 654, 626. MALDI-TOF-MS: $\mathrm{m} / \mathrm{z}=402.76[\mathrm{M}-\mathrm{H}]^{-}$(calcd. For C8H5Br2IO - H = 402.77).

4-chloromandelic acid (p-Cl-MA). White solid. Yield 76\%. Mp. 120.5-122.0 ${ }^{\circ} \mathrm{C} .{ }^{1} \mathrm{H}$ NMR (300 $\mathrm{MHz}_{\mathrm{CDCl}}$ ): $\delta(\mathrm{ppm}) 7.40(\mathrm{~d}, J=8.7 \mathrm{~Hz}, 2 \mathrm{H}), 7.37$ (d, $\left.J=8.7 \mathrm{~Hz}, 2 \mathrm{H}\right), 5.24(\mathrm{~s}, 1 \mathrm{H}) . \mathrm{IR}(\mathrm{KBr}): v$ $\left(\mathrm{cm}^{-1}\right)$ 3411, 2964, 1725, 1490, 1260, 1189, 1058.

2-chloromandelic acid (o-Cl-MA). Pale yellow solid. Yield 80\%. Mp. 86-88 ${ }^{\circ} \mathrm{C} .{ }^{1} \mathrm{H}$ NMR (300 $\left.\mathrm{MHz}, \mathrm{CDCl}_{3}\right): \delta(\mathrm{ppm})$ 7.46-7.39 (m, 2H), 7.34-7.28 (m, 2H), 5.67 (s, 1H). IR (KBr): v (cm $\left.{ }^{-1}\right) 3195$, $1751,1694,1477,1218,1072$.

3-chloromandelic acid (m-Cl-MA). White solid. Yield 51\%. Mp. 117-120 ${ }^{\circ} \mathrm{C} .{ }^{1} \mathrm{H}$ NMR (300 MHz, $\left.\mathrm{CDCl}_{3}\right): \delta(\mathrm{ppm})$ 7.49-7.47 (m, 1H), 7.38-7.32 (m, 3H), 5.24 (s, 1H). IR (KBr): $v\left(\mathrm{~cm}^{-1}\right)$ 3442, 2936, $1712,1577,1261,1195,1063$.

3-bromomandelic acid (m-Br-MA). White solid. Yield 74\%. Mp. 123.5-124.5 ${ }^{\circ} \mathrm{C} .{ }^{1} \mathrm{H}$ NMR (300 $\mathrm{MHz}_{\mathrm{CDCl}}$ ): $\delta(\mathrm{ppm}) 7.64(\mathrm{~s}, 1 \mathrm{H}), 7.47-7.39(\mathrm{~m}, 2 \mathrm{H}), 7.24\left(\mathrm{dd}, J_{1}=J_{2}=7.8 \mathrm{~Hz}, 1 \mathrm{H}\right), 5.12(\mathrm{~s}, 1 \mathrm{H})$. IR (KBr): $v\left(\mathrm{~cm}^{-1}\right)$ 3443, 2933, 1710, 1572, 1474, 1260, 1191, 1097.

3-iodomandelic acid (m-I-MA). White solid. Yield 55\%. Mp. 98-101 ${ }^{\circ} \mathrm{C} .{ }^{1} \mathrm{H}$ NMR (300 MHz, $\left.\mathrm{CDCl}_{3} / \mathrm{CD}_{3} \mathrm{OD}\right): \delta(\mathrm{ppm}) 7.84(\mathrm{~s}, 1 \mathrm{H}), 7.65(\mathrm{~d}, J=7.8 \mathrm{~Hz}, 1 \mathrm{H}), 7.45(\mathrm{~d}, J=8.1 \mathrm{~Hz}, 1 \mathrm{H}), 7.14-7.07$ (m, 1H), 5.09 (s, 1H). IR (KBr): $v\left(\mathrm{~cm}^{-1}\right)$ 3390, 2928, 1739, 1287, 1179, 1106, 940, 780, 748, 685. 
4-fluoromandelic acid (p-F-MA). To the cooled solution of lithium chloride (3.40 g, $80.2 \mathrm{mmol})$ and potassium hydroxide $(9.02 \mathrm{~g}, 161 \mathrm{mmol})$ in water $(32 \mathrm{~mL})$ was added 1,4-dioxane $(32 \mathrm{~mL})$. To the solution were added dropwise 4-fluorobenzaldehyde $(4.97 \mathrm{~g}, 40.1 \mathrm{mmol})$ and bromoform $(10.1 \mathrm{~g}$, $40.1 \mathrm{mmol})$. The mixture was stirred for $19 \mathrm{~h}$ at $5-10{ }^{\circ} \mathrm{C}$ and further $24 \mathrm{~h}$ at room temperature. The mixture was diluted with water and washed with ether $(20 \mathrm{~mL} \times 3)$ and the aqueous phase was acidified with $6 \mathrm{~N} \mathrm{HCl}$ aq. $(50 \mathrm{~mL})$. The aqueous phase was extracted with ether $(50 \mathrm{~mL} \times 10)$ and the combined organic phase was dried over anhydrous sodium sulfate. After concentration under reduced pressure, the residue was dissolved in methanol and benzylamine ( $3.98 \mathrm{~g}, 36.8 \mathrm{mmol})$ was added. After the solvent was removed under reduced pressure, the solid was recrystallized from $80 \%$ ethanol $(69 \mathrm{~mL})$. The obtained salt was added to $1 \mathrm{~N} \mathrm{HCl}$ aq. and extracted with ether $(10 \mathrm{~mL} \times 3)$. The combined organic phase was dried over anhydrous sodium sulfate and the solvent was removed under reduced pressure to give 4-fluoromandelic acid (5.21 g, $30.6 \mathrm{mmol}, 76 \%)$ as a white solid. Mp. 130-132 ${ }^{\circ} \mathrm{C} .{ }^{1} \mathrm{H}$ NMR $\left(300 \mathrm{MHz}, \mathrm{CDCl}_{3}\right): \delta(\mathrm{ppm})$ 7.46-7.42 (m, 2H), 7.10-7.05 (m, 2H), 5.25 (s, 1H). IR (KBr): $v\left(\mathrm{~cm}^{-1}\right)$ 3453, 2911, 1722, 1609, 1519, 1240, 1067.

\section{Enantioseparation experiments}

Racemic mandelic acid $(1 \mathrm{mmol})$ and $(1 R, 2 S)$-ADPE $(1 \mathrm{mmol})$ were dissolved in methanol. After concentration, the resulting white solids were recrystallized from an appropriate solvent to obtain the salt crystals, which were filtered and dried overnight. The apparent yield was calculated based on the ${ }^{1} \mathrm{H}$ NMR data, considering the amount of the included solvent. A portion of the salt was decomposed by the addition of $1 \mathrm{M}$ aqueous $\mathrm{HCl}$ solution and the aqueous layer was extracted with diethyl ether. After drying over anhydrous $\mathrm{Na}_{2} \mathrm{SO}_{4}$, the organic layer was concentrated to obtain enantio-enriched MA. To a solution of MA in toluene $(2 \mathrm{ml})$ and methanol $(1 \mathrm{ml})$ was added $\mathrm{TMSCHN}_{2}$ (ether solution) until the solution remained yellow. The mixture was stirred at room temperature and the solvent was removed under reduced pressure. The residue was purified by silica gel PTLC (hexane/ethyl acetate $=2: 1$ ) to produce methyl ester of MA as a colorless liquid. The ee value of the ester was determined by chiral HPLC analysis.

Methyl ester of $p$-Br-MA: Daicel Chiralcel OD-3, hexane/2-propanol=90:10, $1.0 \mathrm{~mL} / \mathrm{min} ; t_{\mathrm{r}}(S)=$ $8.1 \min , t_{\mathrm{r}}(R)=9.0 \mathrm{~min}$.

Methyl ester of $p$-Cl-MA: Daicel Chiralcel OD-3, hexane/2-propanol=90:10, $1.0 \mathrm{~mL} / \mathrm{min} ; t_{\mathrm{r}}(S)=$ $7.4 \min , t_{\mathrm{r}}(R)=8.5 \mathrm{~min}$.

Methyl ester of $p$-F-MA: Daicel Chiralcel OD-3, hexane/2-propanol=90:10, $1.0 \mathrm{~mL} / \mathrm{min} ; t_{\mathrm{r}}(S)=7.6$ $\min , t_{\mathrm{r}}(R)=9.2 \mathrm{~min}$.

Methyl ester of $m$-Cl-MA: Daicel Chiralcel OD-3, hexane/2-propanol=90:10, $1.0 \mathrm{~mL} / \mathrm{min} ; t_{\mathrm{r}}(S)=$ $7.3 \min , t_{\mathrm{r}}(R)=8.6 \mathrm{~min}$.

Methyl ester of $m$-Br-MA: Daicel Chiralpak IB-3, hexane/2-propanol=90:10, $0.5 \mathrm{~mL} / \mathrm{min} ; t_{\mathrm{r}}(S)=$ 
$15.0 \min , t_{\mathrm{r}}(R)=16.2 \min$.

Methyl ester of $m$-I-MA: Daicel Chiralcel OD-3, hexane/2-propanol=90:10, $1.0 \mathrm{~mL} / \mathrm{min} ; t_{\mathrm{r}}(S)=8.3$ $\min , t_{\mathrm{r}}(R)=10.0 \min$.

\section{X-ray crystallographic analysis}

Single crystals suitable for X-ray diffraction analysis were prepared by slow evaporation of the saturated solutions of the salts. X-ray crystallographic data were collected on a Bruker Smart APEX II diffractometer with graphite monochromated Mo K $\alpha$ radiation. Data collections were carried out at low temperatures $(150 \mathrm{~K})$. The structures were solved by a direct method (SIR 2014) and refined by SHELXL-2013 programs. Crystallographic information files have been deposited with the Cambridge Structural Database.

\section{References}

1) Klingenberg, J. J. Org. Synth. 1955, 35, 11-14.

2) Compere, E. L. J. Org. Chem. 1968, 33, 2565-2566. 